\title{
Universal scaling effects of a temperature gradient at first-order transitions
}

\author{
Claudio Bonati, Massimo D'Elia, Ettore Vicari \\ Dip. di Fisica dell'Università di Pisa and INFN, Largo Pontecorvo 3, I-56127 Pisa, Italy
}

(Dated: February 26, 2018)

\begin{abstract}
We study the effects of smooth inhomogeneities at first-order transitions. We show that a temperature gradient at a thermally-driven first-order transition gives rise to nontrivial universal scaling behaviors with respect to the length scale $l_{t}$ of the variation of the local temperature $T_{x}$. We propose a scaling ansatz to describe the crossover region at the surface where $T_{x}=T_{c}$, where the typical discontinuities of a first-order transition are smoothed out.

The predictions of this scaling theory are checked, and get strongly supported, by numerical results for the 2D Potts models, for a sufficiently large number of states in order to have first-order transitions. Comparing with analogous results at the $2 \mathrm{D}$ Ising transition, we note that the scaling behaviors induced by a smooth inhomogeneity appear quite similar in first-order and continuous transitions.

PACS numbers: 05.70.Fh,05.70.Jk,64.60.De,05.10.Cc
\end{abstract}

\section{INTRODUCTION}

The theory of phase transitions [1, 2] generally applies to homogenous systems. However, homogeneity is often an ideal limit of experimental conditions. Inhomogenous conditions may significantly affect the experimental data at phase transitions, requiring an understanding of their effects for a correct interpretation. In particular, they generally smooth out the singularities of the thermodynamic quantities at phase transitions. Notable examples are temperature gradient effects in general setups (see e.g. $[3-6]$ ), the gravity effects in experimental studies of fluids [7] (in particular at the superfluid transition in ${ }^{4}$ He systems [8]) and in the phenomenology of dense astrophysical objects [9, 10], the external confining forces in cold atom experiments [11], and the intrinsic spacetime inhomogeneity of the quark-gluon plasma formation in heavy-ion collisions [13, 14]. However, inhomogeneity effects should not only be considered as a drawback of the experimental setup, but they may also give rise to interesting peculiar phenomena at phase transitions.

In the presence of smooth inhomogeneities, we may simultaneously observe different phases at different space regions; for example experiments of cold atoms in optical lattices show the simultaneous presence of Mott incompressible and superfluid phases in different regions of the inhomogenous harmonic trap [11]. In noncritical regimes, away from phase transitions when correlations do not develop long length scales, inhomogeneity effects may be effectively taken into account by local-equilibrium approximations (LEA), assuming a local equilibrium analogous to that of the homogenous system at the same thermodynamic parameters. An example is the local-density approximation widely used to study particle systems with an effective space-dependent chemical potential [11, 12]. At continuous (classical or quantum) transitions, where correlations develop large length scales, LEA cannot provide a satisfactory description: critical modes get significantly distorted by the inhomogeneities, which give generally rise to a further length scale $\ell$. However, for sufficiently smooth inhomogeneities we may still observe a peculiar universal scaling with respect to their length scale $\ell$, controlled by the universality class of the transition of the homogenous system [15 17].

In this paper we study the effects of smooth inhomogeneities at first-order transitions, for which little is known. First-order transitions do not develop diverging length scales in the thermodynamic limit, thus one may naively expect trivial behaviors under smooth inhomogenous conditions, describable by LEA. Instead, as we shall see, a nontrivial scaling behavior arises even at first-order transitions, quite similar to that expected at continuous transitions, and controlled by universal critical exponents. In particular, a temperature gradient at a thermal first-order transition gives rise to a nontrivial scaling behavior with respect to its length scale $l_{t} \sim T_{x}\left|\nabla_{x} T_{x}\right|^{-1}$. We study the universal aspects of this scaling, which manifests themselves near $T_{x} \approx T_{c}$ when $l_{t}$ is large (in the infinite volume limit), the typical singularities of first order transitions emerging as $l_{t} \rightarrow \infty$.

The paper is organized as follows. In Sec. II we present the model that we consider as theoretical laboratory where we develop our scaling theory, i.e. the $2 \mathrm{D}$ Potts model in the presence of a temperature gradient. In Sec. III we look at the behavior around the region corresponding to the first-order transition, and compare it with the corresponding LEA, showing how the latter fails to describe the crossover between the two phases. In Sec. IV we put forward scaling ansatzes to describe this behavior across first-order transitions, which extend analogous scaling phenomena expected at continuous transitions. In Sec. $\mathrm{V}$ we present a numerical analysis of the 2D Potts model which supports our scaling theory. Finally, in Sec. VI we draw our conclusions. In App. A we report an analogous numerical analysis for the continuous transition of the 2D Ising model in the presence of a temperature gradient. 


\section{THE MODEL}

For the sake of demonstration, as simple statistical models undergoing first-order transitions, we consider the two-dimensional (2D) $q$-state Potts models for which several exact results are known, such as the critical temperature, the latent heat, etc. [18]. We consider inhomogenous Potts models defined by the partition function

$$
Z=\sum_{\left\{s_{\mathbf{r}}\right\}} e^{-H}, \quad H=-\sum_{i, \hat{\mu}} \frac{J_{\mathbf{r}_{i}}}{2} \delta\left(s_{\mathbf{r}_{i}}, s_{\mathbf{r}_{i}+\hat{\mu}}\right),
$$

where $\mathbf{r}_{i}$ are the sites of a square lattice, $s_{\mathbf{r}_{i}}$ are integer variables $1 \leq s_{\mathbf{r}_{i}} \leq q, \delta(a, b)=1$ if $a=b$ and zero otherwise, and $\hat{\mu}$ denotes denotes the unit vectors along the $x$ and $y$ axes with positive and negative directions. We assume that $J_{\mathbf{r}}$ depends on the position, thus mimicking an effective space-dependent inverse temperature $J_{\mathbf{r}}=T_{\mathbf{r}}^{-1}$. In the homogenous case, i.e. $J_{\mathbf{r}} \equiv J$, the square-lattice Potts model undergoes a phase transition at [19] $J_{c}=T_{c}^{-1}=\ln (1+\sqrt{q})$, which is continuous for $q \leq 4$ and first order for $q>4$. For $q=2$ the Potts model becomes equivalent to the Ising model.

In our study we consider anisotropic lattices with $-L_{x}-1 \leq x \leq L_{x}+1$ and $1 \leq y \leq L_{y}$, and assume translation invariance along the $y$ direction, thus $J_{\mathbf{r}} \equiv J_{x}$. We consider a power-law space dependence:

$$
T_{x}=J_{x}^{-1}=T_{c}\left(1+\frac{x|x|^{p-1}}{l_{t}^{p}}\right), \quad\left[l_{t}>L_{x}, p \geq 1\right]
$$

so that $\Delta T_{x} \equiv T_{x}-T_{c}$ changes sign passing from the high$T$ disordered phase $(x>0)$ to the low- $T$ ordered phase $(x<0) . l_{t}$ provides the length scale of the space variation of $T_{x}$. Of course, the most interesting case is the linear variation, i.e. $p=1$. In this case no a priori knowledge of the $T_{c}$ value is required. However we consider also generic $p>1$ to crosscheck the theoretical predictions. We choose periodic boundary conditions along $y$, and set $s_{-L_{x}-1, y}=1$ and $T_{L_{x}+1}=\infty$. Note that in the $p \rightarrow \infty$ limit with $l_{t}>L_{x}$ we recover a homogenous model in a slab $2 L_{x} \times \infty$ with mixed boundaries, i.e. fixed and disordered.

We are interested in the infinite-volume limit so that only the length scale $l_{t}$ is left. Thus we choose $L_{x}$ and $L_{y}$ sufficiently large to make finite-size effects negligible in the region of interest, around $x=0$.

We want to understand what happens across the firstorder transition, around $x=0$. For this purpose we consider the wall energy density and magnetization

$$
\begin{aligned}
& e(x)=-\frac{\sum_{y}\langle\mathcal{E}(x, y)\rangle}{L_{y}}, \quad \mathcal{E}(x, y)=\delta\left(s_{x, y}, s_{x, y+1}\right),(3) \\
& m(x)=\frac{\sum_{y}\left\langle\mathcal{M}_{1}(x, y)\right\rangle}{L_{y}}, \quad \mathcal{M}_{n}(\mathbf{x})=\frac{q \delta\left(s_{\mathbf{x}}, n\right)-1}{q-1} .
\end{aligned}
$$

In homogenous systems $e(x)$ and $m(x)$ equal the half energy density $E / 2 \equiv\langle H\rangle /(2 J V)$ and the magnetization
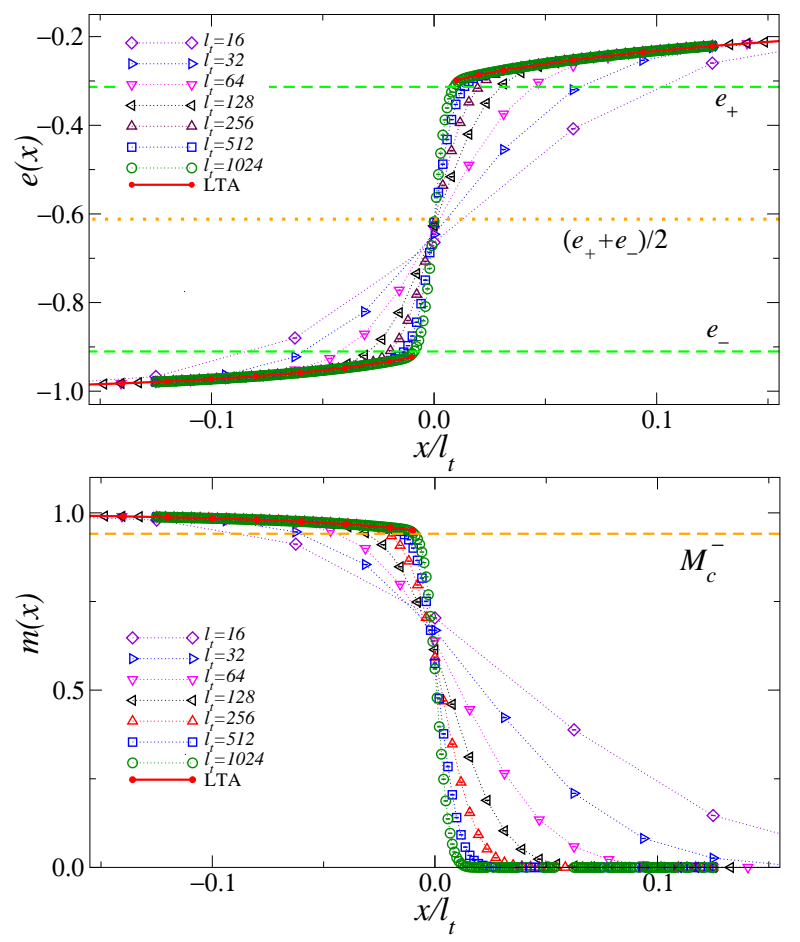

FIG. 1: (Color online) The wall energy density $e(x)$ and magnetization $m(x)$, defined in Eq. (3), for $q=20$ in the case of a linear variation of $T_{x}$, i.e. $p=1$ in Eq. (2), for several values of $l_{t}$. The dashed lines represent the constants $e_{ \pm} \equiv E_{c}^{ \pm} / 2$ (top figure) and $M_{c}^{-}$(bottom figure). The LTA data are hardly distinguishable from the large- $l_{t}$ data.

$M$, respectively. We also consider wall-wall connected correlations

$$
\begin{aligned}
& P_{e}\left(x_{1}, x_{2}\right)=\frac{1}{L_{y}} \sum_{y_{1}, y_{2}}\left\langle\mathcal{E}\left(x_{1}, y_{1}\right) \mathcal{E}\left(x_{2}, y_{2}\right)\right\rangle_{c} \\
& P_{m}\left(x_{1}, x_{2}\right)=\frac{1}{L_{y}} \sum_{y_{1}, y_{2}}\left\langle\mathcal{M}_{1}\left(x_{1}, y_{1}\right) \mathcal{M}_{1}\left(x_{2}, y_{2}\right)\right\rangle_{c} .
\end{aligned}
$$

\section{BEHAVIOR ACROSS THE TRANSITION REGION AND LOCAL TEMPERATURE APPROXIMATION}

We present numerical results for $q=20$, for which, beside $T_{c}$, we exactly know the energy densities $E_{c}^{-} \approx$ $-1.820684, E_{c}^{+} \approx-0.626529$, and the magnetization $M_{c}^{-} \approx 0.9411759$ at $T_{c}^{ \pm}$[18]. Monte Carlo (MC) simulations of the model (1) are performed using a Metropolis algorithm to update the site variables, up to length scales $l_{t}=O\left(10^{3}\right)$.

We check the convergence of the results with respect to the lattice sizes $L_{x}$ and $L_{y}$, so that all data around $x=0$ that we present should be considered as infinitevolume results. In particular, for $p=1$, we checked that $l_{t} / L_{x}=2,4$ and $L_{y} / L_{x}=10,20$ do not give appreciable differences (within the errors) for any observable 
considered, independently of the combination of their choice. In the case $p=2$ we mostly use $l_{t} / L_{x}=2$ and $L_{y} / L_{x}=10,20$. Note that finite-size effects around $x=0$ should be generally controlled by the ratio $L_{\#} / l_{t}^{\theta}$, because $\xi \sim l_{t}^{\theta}$ in the crossover region (the $\theta$ exponent will be defined in the following).

Figure 1 shows MC data of $e(x)$ and $m(x)$ in the case of a linear $T_{x}$. For any $l_{t}$ they vary from the low$T(x<0)$ to the high- $T(x>0)$ regimes, showing a crossover around $x=0$. With increasing $l_{t}$, the data appear to reconstruct the discontinuities of the first-order transition of the homogenous system.

We compare the MC results with a local-temperature approximation (LTA), which estimates $e(x)$ and $m(x)$ using the corresponding $E(T) / 2$ and $M(T)$ of the homogenous system in the thermodynamic limit, i.e.

$$
e(x) \approx e_{\mathrm{lta}}\left(x / l_{t}\right)=E\left[T_{x}\left(x / l_{t}\right)\right] / 2,
$$

and $m(x) \approx m_{\mathrm{lta}}\left(x / l_{t}\right)=M\left[T_{x}\left(x / l_{t}\right)\right]$. Of course, $e_{\mathrm{lta}}(x)$ and $m_{\text {lta }}(x)$ are not defined at $x=0$, because they are discontinuous at $T_{c}$. LTA is expected to provide a good approximation when $T_{x}$ varies smoothly, thus for large $l_{t}$. Since $E(T)$ and $M(T)$ are not known for $T \neq T_{c}$, we compute them by MC simulations of the homogenous system. These LTA results are also shown in Fig. 1.

The MC results show that $e(x)$ and $m(x)$ approach their LTA when we consider the limit $l_{t} \rightarrow \infty$ keeping the ratio $x / l_{t}$ fixed, i.e. at fixed $T_{x}$. The convergence to LTA is fast far from $x=0$ while it becomes significantly slower when approaching $x=0$. As we shall see, this reflects a hidden nontrivial scaling behavior which characterizes the crossover region around $x=0$ in the smooth (i.e. large $l_{t}$ ) limit. This is a novel regime probing the mixed phase at first-order transitions, where $e_{-}<e(x)<e_{+}$ $\left(e_{ \pm} \equiv E_{c}^{ \pm} / 2\right)$ and $0<m(x)<M_{c}^{-}$.

\section{SCALING ANSATZES}

In order to construct a theory which allows us to predict the scaling behavior in this crossover region, we first outline the main features of the scaling behavior at continuous transitions in analogous conditions, such as the 2D Potts model (11) for $q \leq 4$. We assume a standard continuous transition with two relevant parameters: $t \equiv T / T_{c}-1$ and $h$ coupled to the order parameter. The critical behavior is determined by their RG dimensions: for a generic $d$-dimensional system $y_{t} \equiv 1 / \nu$ and $y_{h}=(d+2-\eta) / 2$, where $\nu$ and $\eta$ are the correlationlength and two-point function critical exponents [20]. For example, $\nu=1$ and $\eta=1 / 4$ in the case of the $2 \mathrm{D}$ Ising model. In the presence of an effective $T_{x}$ varying as in Eq. (2), the behavior around the surface $x=0$ where $T_{x}=T_{c}$ can be inferred using scaling arguments such as those reported in Refs. 7, [15]. Some details of their derivation are reported in Appendix A. Extending to generic $(d-1)$-dimensional walls the definitions of the

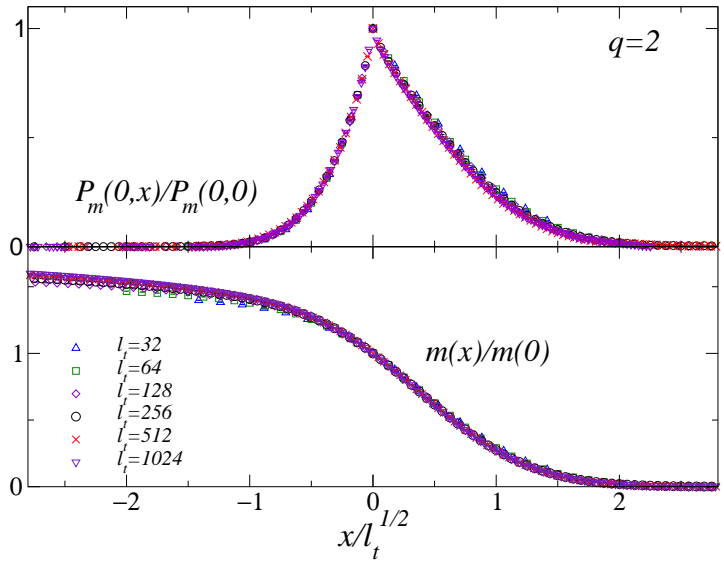

FIG. 2: (Color online) Scaling of $m(x)$ and $P_{m}(0, x)$, defined in Eqs. (3) and (4) respectively, at the continuous Ising transition (i.e. $q=2$ ) for a linear $T_{x}$. The data collapse toward an asymptotic curve when they are plotted versus $x / l_{t}^{\theta}$ with $\theta=1 / 2$. The data at $x=0$ show the power-law behaviors $m(0) \sim l_{t}^{-1 / 16}$ and $P_{m}(0,0) \sim l_{t}^{3 / 8}$, in agreement with the Eqs. (6) and (8).

observables (34 (4), we obtain the large- $l_{t}$ asymptotic behaviors

$$
\begin{aligned}
& m(x) \approx l_{t}^{-\theta\left(d-y_{h}\right)} f_{m}\left(x / l_{t}^{\theta}\right), \\
& e(x) \approx e_{b}\left(x / l_{t}\right)+l_{t}^{-\theta\left(d-y_{t}\right)} f_{e}\left(x / l_{t}^{\theta}\right), \\
& P_{m}\left(x_{1}, x_{2}\right) \approx l_{t}^{\theta\left(1-2 d+2 y_{h}\right)} g_{m}\left(x_{1} / l_{t}^{\theta}, x_{2} / l_{t}^{\theta}\right), \\
& P_{e}\left(x_{1}, x_{2}\right) \approx l_{t}^{\theta\left(1-2 d+2 y_{t}\right)} g_{e}\left(x_{1} / l_{t}^{\theta}, x_{2} / l_{t}^{\theta}\right), \\
& \theta=p /\left(p+y_{t}\right) \leq 1,
\end{aligned}
$$

where we used the fact that the power-law scaling is essentially determined by the RG dimensions of the energy density, $y_{e}=d-y_{t}$, and the magnetization, $y_{m}=d-y_{h}$. The exponent $\theta$ tells us how to rescale the distances around $x=0$ to get a nontrivial scaling behavior, thus implying that the length scale $\xi$ of the critical modes behaves as $\xi \sim l_{t}^{\theta}$. The term $e_{b}\left(x / l_{t}\right)$ in the r.h.s. of Eq. (7) is a background contribution, like that appearing in homogenous systems [20, 21]; in Appendix $₫$ we argue that it coincides with the LTA of the energy. The approach to the asymptotic behaviors is characterized by $O\left(l_{t}^{-\theta}\right)$ corrections with respect to the leading terms. Note that the above scaling behavior has some analogies with the Finite Size Scaling (FSS) theory for homogenous systems of size $L^{d}$ [22], with three main differences: the system is effectively infinite, the correlation length $\xi$ has a nontrivial power-law dependence on $l_{t}$ (instead of $\xi \sim L$ ) and the spatial inhomogeneity of the system. Fig. 2 shows some results for $q=2$ with a linear $T_{x}$, i.e. $p=1$ in Eq. (2). They definitely support the scaling behaviors predicted by Eqs. (6) and (8).

Let us now go back to first-order transitions, focussing on the crossover region around $x=0$, see Fig. 1 Our working hypothesis is that the analogy of the inhomogenous scaling ansatz (6) and (7) with the FSS of the ho- 
mogenous system may also extend to first-order transitions. Moreover, we recall that the FSS at a first-order transition turns out to be similar to that at continuous transitions, being characterized by extreme RG dimensions [23 25] $y_{t}=y_{h}=d$ (corresponding to $\nu=1 / d$ and $\eta=2-d)$. Then, it is natural to conjecture that the crossover region around $x=0$ at first-order transitions is also described by the scaling behavior at continuous transitions, replacing $y_{t}=y_{h}=d$ in Eqs. (6-10). This leads to the scaling behaviors

$$
\begin{array}{ll}
e(x) \approx f_{e}\left(x / l_{t}^{\theta}\right), \quad & P_{e}\left(x_{i}\right) \approx l_{t}^{\theta} g_{e}\left(x_{i} / l_{t}^{\theta}\right), \\
m(x) \approx f_{m}\left(x / l_{t}^{\theta}\right), & P_{m}\left(x_{i}\right) \approx l_{t}^{\theta} g_{m}\left(x_{i} / l_{t}^{\theta}\right), \\
\theta=p /(d+p) \leq 1 . &
\end{array}
$$

Unlike Eq. (7), we do not expect background terms in the wall energy density due to the fact that its LTA does not take values between $e_{-} \equiv E_{c}^{-} / 2$ and $e_{+} \equiv E_{c}^{+} / 2$. Our scaling conjecture is quite general, i.e. it should apply to any first-order transition with the local temperature dependence (2), and in particular for a linear $T_{x}$ for which $\theta=1 /(d+1)$. Moreover, we expect that the asymptotic behaviors are generally approached with $O\left(l_{t}^{-\theta}\right)$ corrections with respect to the leading term.

An important remark is in order: the inhomogeneous scaling ansatz at first order transitions is by no means a trivial extension of the continuous transition case, since in the $l_{t} \rightarrow \infty$ limit it must reconstruct the peculiar singularity of the first order transition, which is not related to a diverging length scale.

\section{SCALING ACROSS THE FIRST-ORDER TRANSITION REGION IN 2D POTTS MODELS}

In Figs. 3. 4 and 5] we show data for $p=1$ and $p=2$, for which $\theta=1 / 3$ and $\theta=1 / 2$ respectively. The data of the wall energy and magnetization and the ratios $P_{e}(0, x) / P_{e}(0,0)$ and $P_{m}(0, x) / P_{m}(0,0)$ clearly approach asymptotic curves when they are plotted versus $x / l_{t}^{\theta}$, in agreement with the scaling behaviors predicted by Eqs. (11, 13).

Note that a smooth matching with the asymptotic behavior at fixed $x / l_{t}$, approaching LTA, requires $f_{e}( \pm \infty)=e_{ \pm}$, which is also supported by the data, as shown by Figs. 3 and 5. Analogous results are also obtained for $q=10$, whose latent heat is substantially smaller $(\Delta \approx 0.696)$ : the exponents are the same, but the scaling functions in Eqs. (11, 12) differ quantitatively, as expected, although they appear qualitatively similar. It is however important to notice that we expect the weaker the transition the larger $l_{t}$ has to be in order to observe the asymptotic scaling.

The approach of the data to the asymptotic behavior is also consistent with the expected $O\left(l_{t}^{-\theta}\right)$ corrections. In particular, the data of $e(x)$ support the relation $e(0)=\left(e_{+}+e_{-}\right) / 2+O\left(l_{t}^{-\theta}\right)$, thus $f_{e}(0)=\left(e_{+}+e_{-}\right) / 2$.

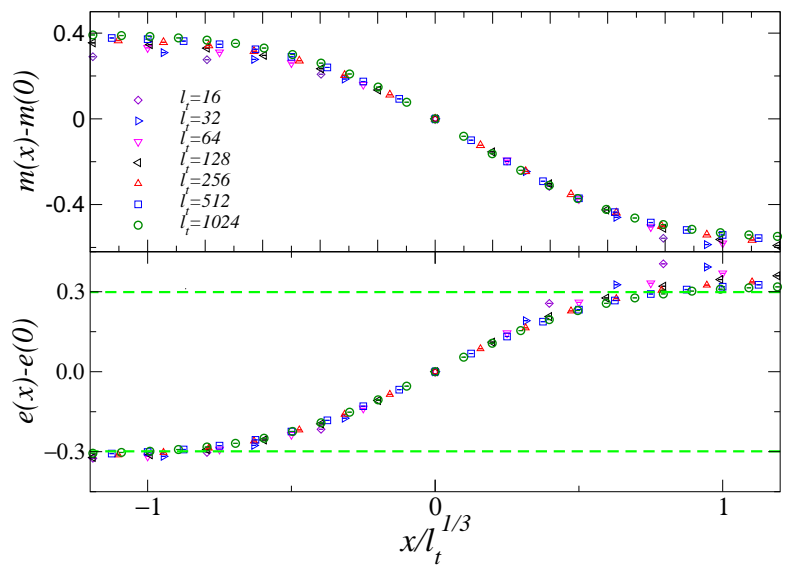

FIG. 3: (Color online) Scaling of the wall energy density and magnetization for $q=20$ with a linear $T_{x}$. We plot the differences $e(x)-e(0)$ (bottom) and $m(x)-m(0)$ (top) which have smaller statistical errors. The data clearly approach asymptotic curves in agreement with the scaling predictions. In the bottom figure the dashed lines show the expected asymptotic values of the scaling curves, which are $f_{e}( \pm \infty)-f_{e}(0)= \pm\left(e_{+}-e_{-}\right) / 2$.
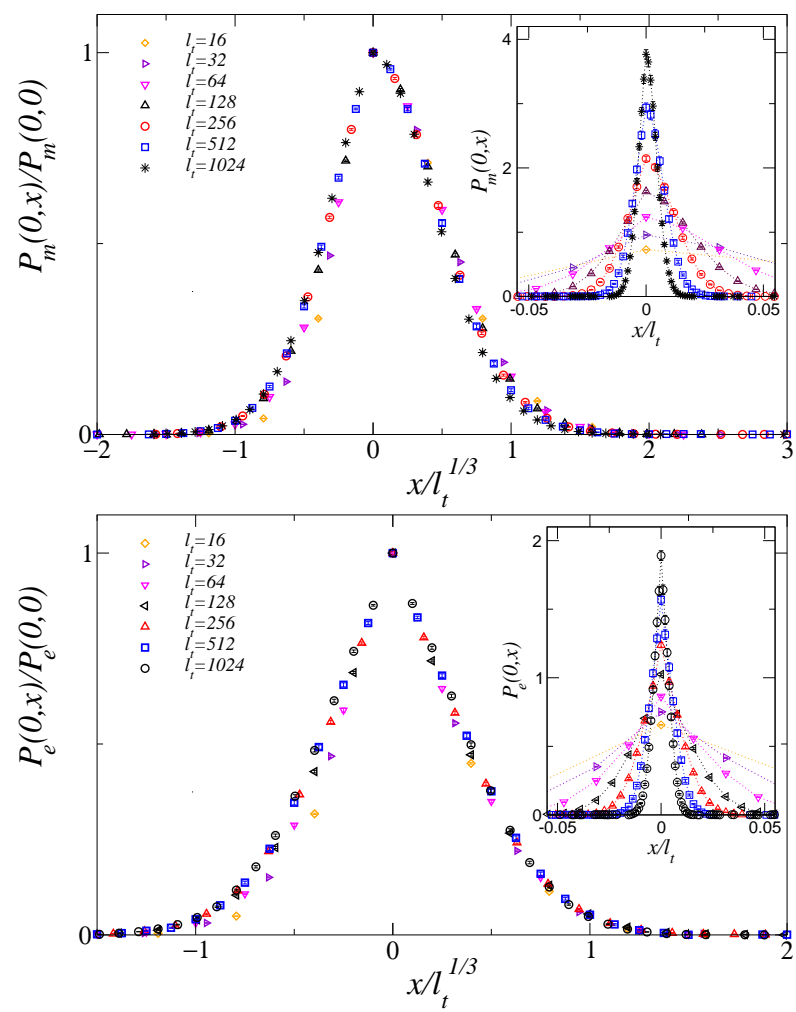

FIG. 4: (Color online) Scaling of the wall-wall correlations $P_{e}(0, x)$ (bottom) and $P_{m}(0, x)$ (top) for $q=20$ with a linear $T_{x}$. The ratios $P_{\#}(0, x) / P_{\#}(0,0)$ versus $x / l_{t}^{\theta}$ (with $\theta=1 / 3$ ) approach scaling curves, in agreement with Eqs. (11:12). The insets show the raw data of $P_{\#}(0, x)$ vs $x / l_{t}$. Note that the data also support the scaling predictions $P_{\#}(0,0) \sim l_{t}^{\theta}$. 


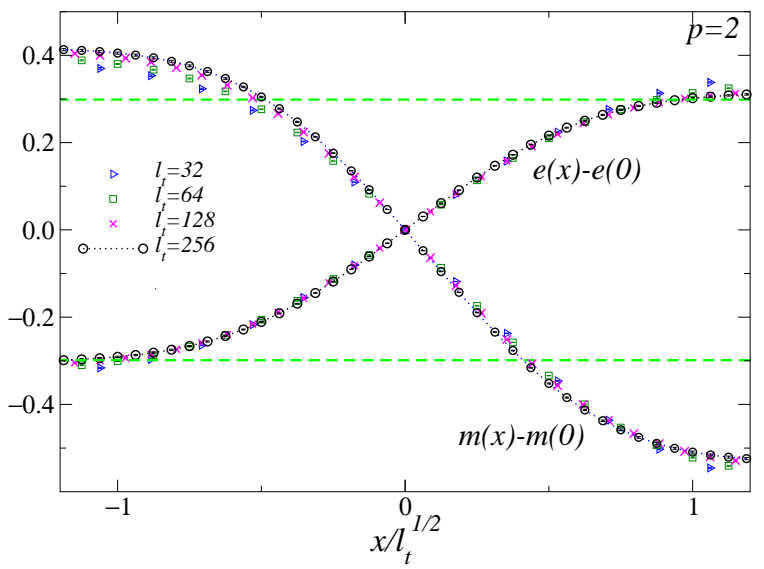

FIG. 5: (Color online) The wall energy density and magnetization for $q=20$ and a quadratic $T_{x}$, i.e. $p=2$ in Eq. (2). The data clearly approach an asymptotic curve $f\left(x / l_{t}^{\theta}\right)$ with increasing $l_{t}$. The dashed lines show the expected asymptotic values of the energy-density scaling curves, which are $f_{e}( \pm \infty)-f_{e}(0)= \pm\left(e_{+}-e_{-}\right) / 2$.

A numerical evidence of this fact can be obtained by analyzing the large- $l_{t}$ behavior of MC data at fixed $x / l_{t}^{\theta}$, and in particular at $x=0$. In Figs. [6 and 7 we show data for the wall energy density and magnetization and the correlation $P_{e}(0, x)$ at $x=0$, in the case of linear and quadratic dependence of the temperature respectively, i.e. $p=1$ and $p=2$, corresponding to $\theta=1 / 3$ and $\theta=1 / 2$ respectively. The inhomogeneous scaling behaviors (6 919) predict that $e(0)$ and $m(0)$ go to a constant, while $P_{e}(0,0) \sim l^{\theta}$. The data are clearly consistent with an $O\left(l_{t}^{-\theta}\right)$ approach to the corresponding $l_{t} \rightarrow \infty$ limit. In particular, the energy density appears to converge to the value $\left(e_{+}+e_{-}\right) / 2$, where $e_{ \pm}=E_{c}^{ \pm} / 2$ and [18] $E_{c}^{-} \approx-1.820684, E_{c}^{+} \approx-0.626529$.

Note that these results imply that the curves for different values of $l_{t}$ cross each other around $x=0$, as shown in Fig. 1 and this crossing point approaches the line at $T_{c}$. One may exploit this property to estimate $T_{c}$ when it is not known, using a linear $T_{x}$ (for which the line $x=0$ is not particular) and looking at the crossing point of the wall energy density and magnetization data. The results are expected to approach $T_{c}$ with corrections corresponding to $\Delta T \equiv T-T_{c}=O\left(l_{t}^{-1}\right)$.

\section{CONCLUSIONS}

We have shown that a temperature gradient $\left|\nabla_{x} T_{x}\right| / T_{x} \sim l_{t}^{-1}$ induces nontrivial universal scaling behaviors at first-order transitions driven by the temperature. We propose a scaling ansatz to describe, in the large $l_{t}$ limit, the crossover region at $T_{x} \approx T_{c}$, where the typical discontinuities of a first-order transition get smoothed out, and the system is effectively probing the mixed phase. This scaling behavior is nontrivial, and it

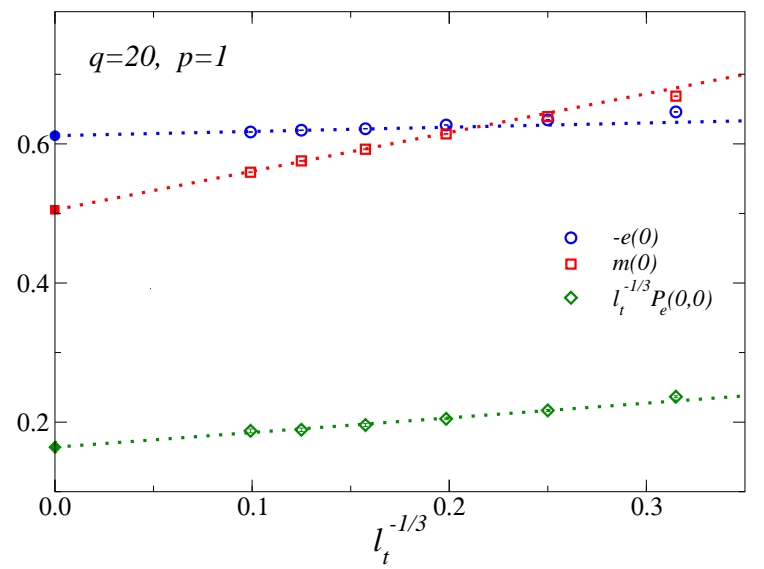

FIG. 6: (Color online) Large- $l_{t}$ behavior at $x=0$ of the wall energy density and magnetization and the correlation $P_{e}$, in the case of a linear variation of the temperature, whose $\theta=1 / 3$. The lines show fits of the data to $a+b l_{t}^{-1 / 3}$.

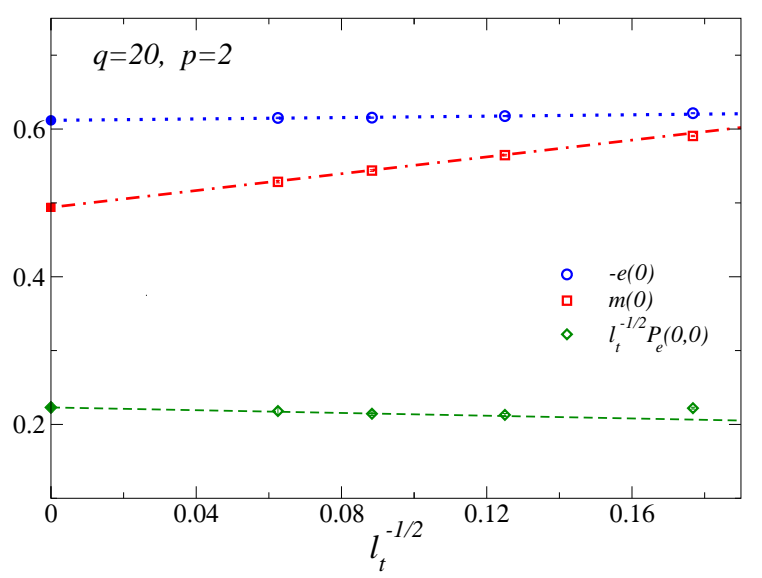

FIG. 7: (Color online) Large- $l_{t}$ behavior at $x=0$ of the wall energy density and magnetization and the correlation $P_{e}$, in the case of a quadratic variation of the temperature, whose $\theta=1 / 2$. The lines show fits of the data to $a+b l_{t}^{-1 / 2}$.

is such that the typical singularities of first-order transitions must be recovered in the limit $l_{t} \rightarrow \infty$. We provide numerical evidence of such phenomenon in the case of the 2D Potts models, for a sufficiently large number of states in order to have first-order transitions. Comparing with analogous results at the 2D Ising transition, we note that the scaling behaviors induced by a smooth inhomogeneity at first-order transitions appear quite similar to that at continuous transitions.

Our approach is quite general, the results can be straightforwardly extended to other sources of inhomogeneities smoothing out the singularities of the transition. For example, an analogous scaling behavior is expected in the case the inhomogeneity arises from an external source coupled to the order parameter (for example, this is the case of the $2 \mathrm{D}$ Ising model in the low- $T$ phase with an inhomogenous magnetic field), or when it entails a 
space-dependent density in particle systems, such as in cold atom experiments. We believe that these peculiar scaling effects of smooth inhomogeneities at first-order transitions should be observable in experiments of physical systems, requiring essentially the possibility of measuring local quantities, and controlling and tuning the length-scale of the inhomogeneity.

Acknowledgement: It is a pleasure to thank Jacopo Nespolo and Paolo Rossi for useful comments and discussions.

\section{Appendix A: Ising model with an inhomogenous temperature}

We report a detailed analysis of the scaling behavior of systems undergoing continuous transitions in inhomogeneous conditions which can be effectively described by a local space-dependent temperature along one direction as in Eq. (2). This analysis can be straightforwardly extended to variations involving more directions.

In order to compute the exponent $\theta$ associated with the temperature inhomogeneity, we need to derive the renormalization-group ( $\mathrm{RG}$ ) properties of the perturbation induced by the external field. For this purpose we may follow the field-theoretical approach of Ref. 21], and consider for simplicity the Ising universality class which can be described by a $d$-dimensional $\Phi^{4}$ quantum field theory

$$
H_{\Phi^{4}}=\int d^{d} x\left[\partial_{\mu} \phi(\mathbf{x})^{2}+r \phi(\mathbf{x})^{2}+u \phi(\mathbf{x})^{4}\right],
$$

where $\phi$ is a real field associated with the order parameter, and $r, u$ are coupling constants. Since the temperature is related to the energy operator $\phi^{2}$, we can write the perturbation $P_{T_{x}}$ as

$$
\begin{aligned}
P_{T_{x}} & =\int d^{d} x t(x) \phi(\mathbf{x})^{2}, \\
t(x) & \equiv \frac{T_{x}-T_{c}}{T_{c}} \sim v^{p} x|x|^{p-1} .
\end{aligned}
$$

Introducing the RG dimension $y_{v}$ of the constant $v$, we derive the $R G$ relation

$$
p y_{v}-p+y_{e}=d,
$$

where $y_{e}=d-1 / \nu$ is the RG dimension of the energy operator. We eventually obtain

$$
\theta=\frac{1}{y_{v}}=\frac{p \nu}{1+p \nu} .
$$

This is equivalent to assuming that $t(x)$ has globally $\mathrm{RG}$ dimension $y_{t}$, thus under a change of length scale $x \rightarrow x / b$ for which $v \rightarrow v_{b}$, it transforms into

$$
b^{y_{t}} v^{p} x|x|^{p-1}=v_{b}^{p} b^{-p} x|x|^{p-1}
$$

so that $v_{b}=b^{1+y_{t} / p} v$, thus implying Eq. A5 .

In particular for a linear variation, i.e. nonzero constant temperature gradient, we have $\theta=\nu /(1+\nu)$. Actually, this result is very general, i.e. it holds for any continuous transition, replacing the appropriate exponent $\nu$. This RG scaling analysis leads to the following singular part of the free energy density

$$
F=l_{t}^{-\theta d} \mathcal{F}\left(t l_{t}^{\theta y_{t}}, h l_{t}^{\theta y_{h}}, x l_{t}^{-\theta}\right) .
$$

This is quite analogous to the scaling of particle systems in a inhomogeneous trap [15, 21]. Note that the above scaling behavior has some analogies with the FSS theory for homogenous systems of size $L^{d}$ [22], with two main differences: the inhomogeneity due to the space-dependence of the external field, and the nontrivial power-law dependence of the correlation length $\xi$ when increasing $l_{t}$, instead of simply $\xi \sim L$.

Then we consider generic observables defined within translationally-invariant walls with coordinate $x$ along the direction where the temperature varies, such as the wall energy density and magnetization defined in Eq. (3). Their asymptotic scaling behaviors for large $l_{t}$ is expected to be

$$
O(x) \approx l_{t}^{-\theta y_{o}} f_{o}\left(x / l_{t}^{\theta}\right),
$$

where $y_{o}$ is the RG dimension of the corresponding local operator $\mathcal{O}$ at the fixed point describing the critical behavior of the homogenous system. For example, the RG dimensions of the order parameter (magnetization) is $y_{m}=d-y_{h}$ and that of the energy density is $y_{e}=d-y_{t}[20]$. Then for the particular observables (3. (4) we obtain the asymptotic behaviors (66-19). Note that in some cases analytic backgrounds arise, beside the scaling behaviors [20, 21], such as the case of the energy density, cf. Eq. (7). The approach to the asymptotic scaling behavior is characterized by relative $O\left(l_{t}^{-\theta}\right)$ corrections, as argued in Ref. 21] for analogous issues.

In the following we focus on the 2D Ising model, which is equivalent to the $q=2$ Potts model (1), with a linear temperature dependence on space, for which we have $\nu=$ $1, \eta=1 / 4$ and $\theta=1 / 2$. An analogous study for the quantum Ising chain was reported in Ref. [26]. We recall that continuous transitions occur also for $q=3,4$. Their critical exponents are $\nu=5 / 6$ and $\eta=4 / 15$ for $q=3$, $\nu=2 / 3$ and $\eta=1 / 4$ for $q=4$. Then, using Eq. (10) one may derive the corresponding $\theta$ exponent, e.g. we obtain respectively $\theta=5 / 11$ and $\theta=2 / 5$ for $p=1$.

In Fig. 8 we show results for the wall energy density and magnetization. We compare these results with the corresponding local-temperature approximation (LTA), i.e.

$$
\begin{aligned}
& e(x) \approx e_{\mathrm{lta}}\left(x / l_{t}\right) \equiv E_{\mathrm{Is}}\left[T_{x}\left(x / l_{t}\right)\right] / 2, \\
& m(x) \approx m_{\mathrm{lta}}\left(x / l_{t}\right) \equiv M_{\mathrm{Is}}\left[T_{x}\left(x / l_{t}\right)\right],
\end{aligned}
$$

where $E_{\mathrm{Is}}(T)$ and $M_{\mathrm{Is}}(T)$ are the energy density and magnetization of the homogenous system at the temperature $T$ in the thermodynamic limit, which are exactly 

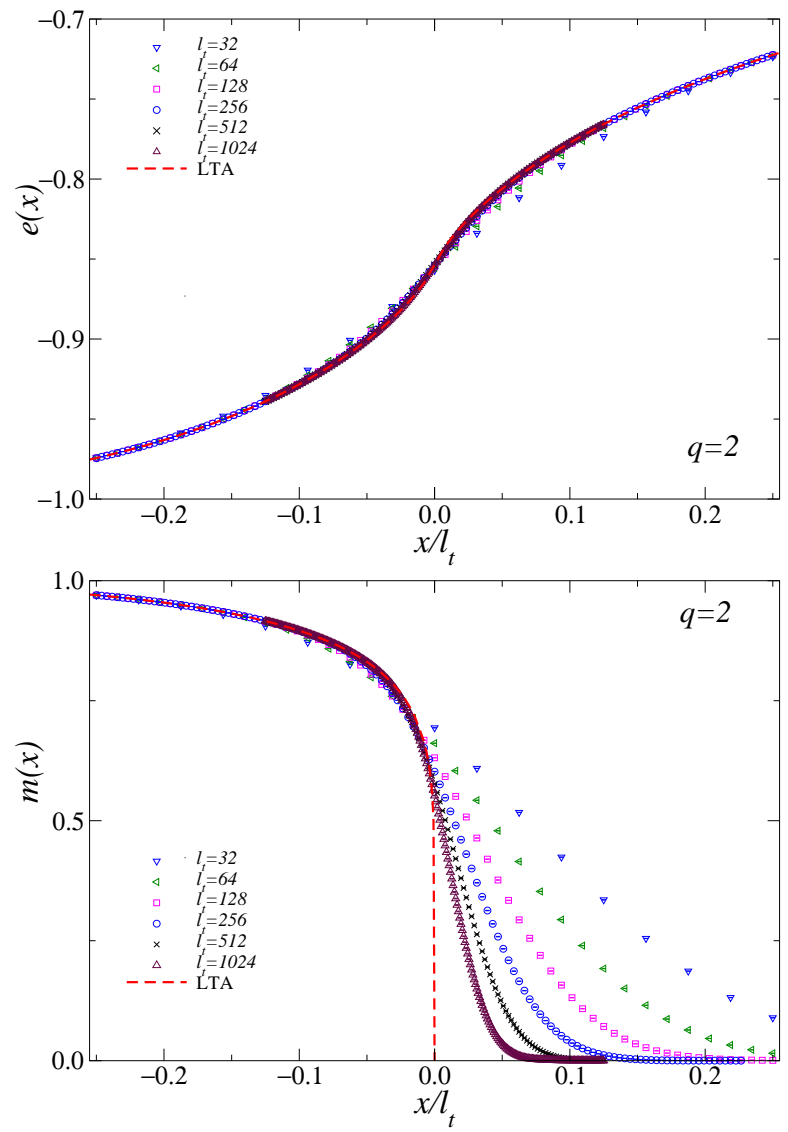

FIG. 8: (Color online) We show data for the wall energy density and magnetization for the $2 \mathrm{D}$ Ising model with a linear $T_{x}$. The dashed lines show the corresponding LTA, cf. Eq. (A9) and (A10), which are hardly distinguishable from the large- $l_{t}$ data.

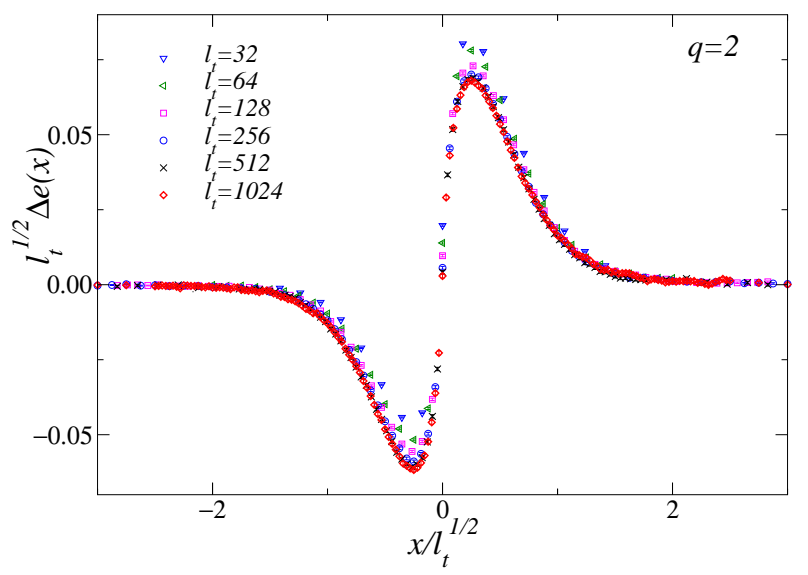

FIG. 9: (Color online) Data for the subtracted wall energy density A15. The data clearly converge toward an asymptotic curve $f_{e}\left(x / l_{t}^{\theta}\right)$, in agreement with the scaling behavior reported in Eq. A16). The data suggest that the scaling function $f_{e}$ is odd.

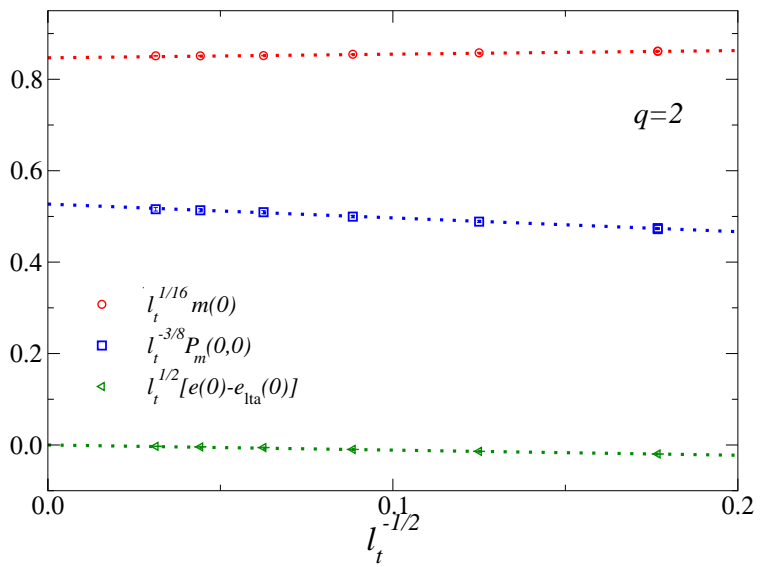

FIG. 10: (Color online) We check the scaling corrections of some quantities at $x=0$ where $T_{x}=T_{c}$. The dotted and dashed lines show fits of the data to $a+b l_{t}^{-1 / 2}$. In particular, in the case of the energy density the data converge to zero, thus implying $f_{e}(0)=0$, as also suggested by the numerical evidence that $f_{e}(x)$ is odd, see Fig. 9

known for the 2D Ising model [27]. Setting

$$
\beta \equiv 1 / T, \quad \tau \equiv \frac{1-\sinh (\beta)^{2}}{2 \sinh (\beta)},
$$

the magnetization in the low- $T$ phase reads

$$
M_{\mathrm{Is}}(T)=\left[1-\left(\sqrt{1+\tau^{2}}+\tau\right)^{2}\right]^{1 / 8},
$$

and the energy density

$$
\begin{aligned}
E_{\mathrm{Is}}(T)= & -\frac{\partial F}{\partial \beta}, \\
F= & \beta+\ln [\sqrt{2} \cosh (\beta)]+ \\
& +\int_{0}^{\pi} \frac{d \varphi}{2 \pi} \ln \left\{1+\left[1-\frac{\cos ^{2} \varphi}{1+\tau^{2}}\right]^{1 / 2}\right\} .
\end{aligned}
$$

The LTA of the energy density and magnetization, cf. Eqs. (A9) and (A10), are expected to improve with increasing $l_{t}$. An educated guess is that LTA provides their $l_{t} \rightarrow \infty$ limit keeping the ratio $x / l_{t}$ fixed. This is confirmed by the data shown in Fig. 8 their convergence appears fast far from $x=0$ coinciding with $T_{c}$, but becomes slower when approaching $x=0$. These larger deviations around $x=0$ reflect the above-discussed scaling behavior characterized by the lenght scale $l_{t}^{\theta}$, which sets in around $x=0$.

The data shown in Fig. 2 nicely confirm the predictions for the magnetization and its correlation. Indeed the data rapidly approach an asympotic curve when they are plotted versus the ratio $x / l_{t}^{\theta}$. Concerning the energy density we consider the subtracted quantity

$$
\Delta e(x) \equiv e(x)-e_{\mathrm{lta}}\left(x / l_{t}\right) .
$$


We argue that

$$
\Delta e(x) \approx l_{t}^{-\theta} f_{e}\left(x / l_{t}^{\theta}\right)
$$

i.e. after subtracting the corresponding LTA only the nontrivial scaling part is left. This is shown by the MC data of the subtracted quantity A15 shown in Fig. 9 This allows us to write the wall energy density as

$$
e(x) \approx e_{\mathrm{lta}}\left(x / l_{t}\right)+l_{t}^{-\theta y_{e}} f_{e}\left(x / l_{t}^{\theta}\right)
$$

Finally, we check the approach to the asymptotic behavior. Fig. 10 shows it for the wall energy density, magnetization and their correlations at $x=0$, confirming the prediction that they are $O\left(l_{t}^{-\theta}\right)$, i.e. $O\left(l_{t}^{-1 / 2}\right)$ in this case.
[1] L. D. Landau and E. M. Lifshitz, Statistical Physics (Pergamon Press, 1969).

[2] K. G. Wilson, in Nobel Lectures in Physics 1981-1990, G. Ekspong Ed., World Scientific Publ., Singapore, 1993; K. G. Wilson and J. Kogut, Phys. Rep. 12, 77 (1974).

[3] J.-P. Brantut, C. Grenier, J. Meineke, D. Stadler, S. Krinner, C. Kollath, T. Esslinger, A. Georges, Science 342, 713 (2013) arXiv:1306.5754 [cond-mat.quant-gas]].

[4] C. J. Takacs, A. Vailati, R. Cerbino, S. Mazzoni, M. Giglio and D. S. Cannell, Phys. Rev. Lett. 106, 244502 (2011).

[5] A. Petkoíc, N. M. Chtchelkatchev, T. I. Baturina and V. M. Vinokur, Phys. Rev. Lett. 105, 187003 (2010) arXiv:1005.4716 [cond-mat.str-el]].

[6] J. Kumaki, T. Hashimoto and S. Granick, Phys. Rev. Lett. 77, 1990 (1996).

[7] M. R. Moldover, J. V. Sengers, R. W. Gammon, and R. J. Hocken, Rev. Mod. Phys. 51, 79 (1979).

[8] J. A. Lipa, D. R. Swanson, J. A. Nissen, T. C. P. Chui, and U. E. Israelsson, Phys. Rev. Lett. 76, 944 (1996).

[9] N. K. Glendenning, Compact Stars (Springer, 2000).

[10] N. Itoh, Prog. Theor. Phys. 44, 291 (1970).

[11] I. Bloch, J. Dalibard, and W. Zwerger, Rev. Mod. Phys. 80, 885 (2008) arXiv:0704.3011 [cond-mat.other]].

[12] S. Giorgini, L. P. Pitaevskii, and S. Stringari, Rev. Mod. Phys. 80, 1215 (2008) arXiv:0706.3360 [cond-mat.other]]

[13] K. H. Ackermann et al. [STAR Collaboration], Phys. Rev. Lett. 86, 402 (2001) nucl-ex/0009011]. K. Aamodt et al. [ALICE Collaboration], Phys. Rev. Lett. 105, 252302 (2010) arXiv:1011.3914 [nucl-ex]].
[14] J. D. Bjorken, Phys. Rev. D 27, 140 (1983).

[15] M. Campostrini and E. Vicari, Phys. Rev. Lett. 102, 240601 (2009); (E) 103, 269901 (2009) arXiv:0903.5153 [cond-mat.stat-mech]].

[16] L. Pollet, N. V. Prokofèv, and B. V. Svistunov, Phys. Rev. Lett. 104, 245705 (2010) arXiv:1003.2655 [condmat.quant-gas]].

[17] Q. Zhou and T.-L. Ho, Phys. Rev. Lett. 105, 245702 (2010) arXiv:1006.1174 [cond-mat.quant-gas]].

[18] F. Y. Wu, Rev. Mod. Phys. 64, 235 (1982).

[19] R. J. Baxter, Exactly solved models in statistical mechanics, (Academic Press, 1982).

[20] A. Pelissetto and E. Vicari, Phys. Rep. 368, 549 (2002) arXiv:cond-mat/0012164 [cond-mat.stat-mech]].

[21] M. Campostrini and E. Vicari, Phys. Rev. A 81, 023606 (2010) arXiv:1003.3334 [cond-mat.stat-mech]].

[22] M. E. Fisher, M. N. Barber, and D. Jasnow, Phys. Rev. A 8, 1111 (1973).

[23] B. Nienhuis and M. Nauenberg, Phys. Rev. Lett. 35, 477 (1975).

[24] M. E. Fisher and A. N. Berker, Phys. Rev. B 26, 2507 (1982).

[25] K. Vollmayr, J. D. Reger, M. Scheucher, and K. Binder, Z. Phys. B 91, 113 (1993).

[26] T. Platini, D. Karevski, and L. Turban, J. Phys. A 40, 1467 (2007) arXiv:cond-mat/0611213 [cond-mat.other]].

[27] B. M. McCoy and T. T. Wu, The two-dimensional Ising model (Cambridge MA, Harward University Press, 1973). 